\title{
The influence of an upstream pylon on open rotor aerodynamics at angle of attack
}

\author{
Nishad G. Sohoni ${ }^{1}$, Cesare A. Hall ${ }^{2 \dagger}$, Anthony B. Parry ${ }^{3}$

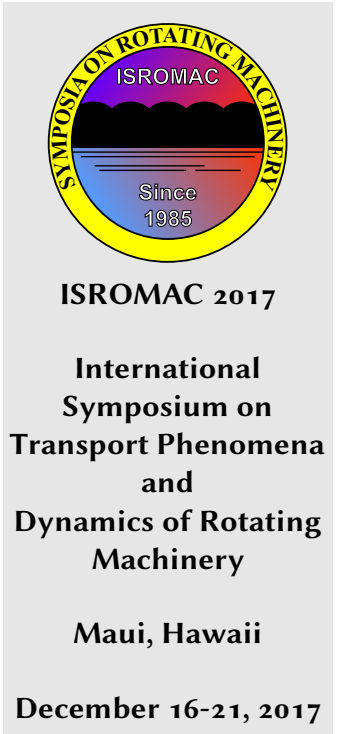

\begin{abstract} a smaller contribution related to change of radius.

Keywords

CROR - pylon - angle-of-attack - pusher - URANS

${ }^{1}$ Whittle Laboratory, University of Cambridge, UK

${ }^{2}$ Whittle Laboratory, University of Cambridge, UK

3 University of Southampton, UK

† Corresponding author: cah1003@cam.ac.uk
\end{abstract}

The aerodynamic impact of installing a horizontal pylon in front of a contra-rotating open rotor engine, at take-off, was studied. The unsteady interactions of the pylon's wake and potential field with the rotor blades were predicted by full-annulus URANS CFD calculations at $0^{\circ}$ and $12^{\circ}$ angle of attack (AoA). Two pylon configurations were studied: one where the front rotor blades move down behind the pylon (DBP), and one where they move up behind the pylon (UBP). When operating at $12^{\circ}$ AoA, the UBP orientation was shown to reduce the rear rotor tip vortex sizes and separated flow regions, whereas the front rotor wake and vortex sizes were increased. In contrast, the DBP orientation was found to reduce the incidence variations onto the front rotor, leading to smaller wakes and vortices. The engine flow was also time-averaged, and the variation in work done on average mid-span streamlines was shown to depend strongly on variation in incidence, along with

\section{NOMENCLATURE}

\author{
Symbols \\ $\alpha_{\text {loc }}$ Local AoA, $\arctan \left(V_{y} / V_{x}\right)$ \\ c Chord \\ $C_{P}$ Power Coefficient, $P /\left(\rho \Omega^{3}(2 R)^{5}\right)$ \\ $C_{T}$ Thrust Coefficient, $T /\left(\rho \Omega^{2}(2 R)^{4}\right)$ \\ D Tip Diameter \\ h Specific enthalpy \\ J Advance Ratio, $V_{\infty} /(2 R \Omega)$ \\ $\mathrm{P}$ Pressure, Power \\ R Tip Radius \\ t Thickness \\ $\mathrm{T}$ Temperature, Thrust \\ V Velocity \\ Z Blade Count \\ $\eta$ Propeller Efficiency, $\left(T V_{\infty}\right) / P$ \\ $\gamma$ Stagger \\ $\Gamma$ Circulation \\ $\Omega$ Rotation speed \\ $\omega$ Vorticity, $\nabla \times V$ \\ $\Phi_{P}$ Pitch Angle \\ $\Phi_{Y}$ Yaw Angle \\ $\rho$ Density \\ $\zeta$ Entropy Loss Coefficient
}

\author{
Subscripts \\ $\infty$ Free-stream \\ o Stagnation \\ F Front Rotor \\ r Radial \\ R Rear Rotor \\ s Static \\ $\theta$ Circumferential \\ $\mathrm{x}$ Axial \\ y Vertical, $\theta=0$
}

\section{INTRODUCTION}

Open rotors enable the use of lower fan pressure ratios, and are therefore potentially more fuel-efficient than turbofans. However, there are challenges related to noise generation that need to be overcome for open rotors to be a viable alternative. In addition to the noise generated by an isolated engine, the installation of a pylon creates additional aerodynamic interactions, which lead to greater noise emission. This pylonrotor interaction is influenced by the engine angle of attack (AoA), which is the angle between the free stream and the engine central axis.

The pylon wake is known to be a significant factor in increased noise, and wake filling has been reported by several projects. Ricouard et al.[1] measured changes in the front rotor blade passing frequency and its harmonics due to pylon trailing edge blowing. The key result from this work was 


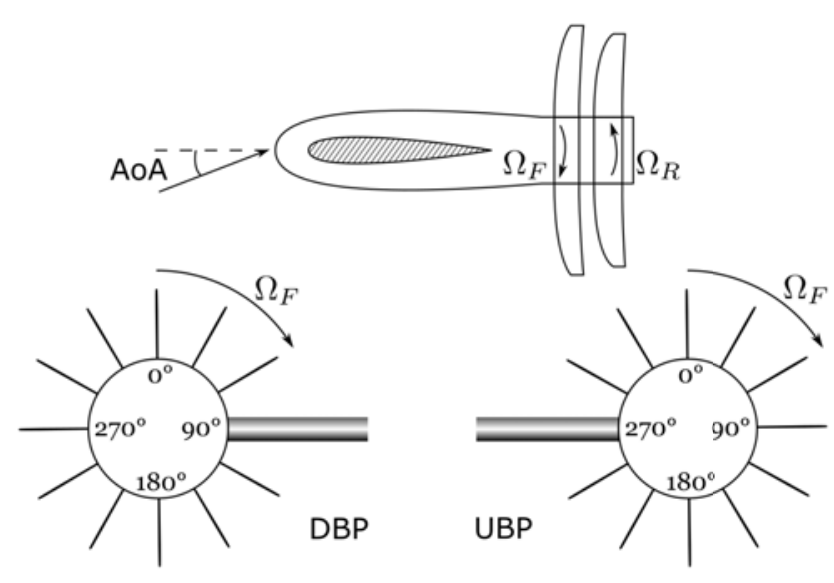

Figure 1. Definition of angle of attack (AoA); and pylon orientation relative to front rotor rotation: Down Behind Pylon (DBP) and Up Behind Pylon (UBP)

that optimum pylon blowing was very effective for reducing rotor-alone tones and broadband noise at $0^{\circ}$ AoA back down to isolated levels. They found, however, that the first four interaction tones were unchanged.

The effect of a pylon on rotor loads has received some attention. Simulations by Boisard et al.[2] at $0^{\circ}$ AoA had a large support strut 2.6 front rotor diameters upstream of the engine, which caused a localised jump in the rotor loads. That is, the force on a blade increased sharply as it passed behind the pylon, then gradually decreased as it continued around the annulus. On the front rotor, this jump occurred over roughly $30^{\circ}$ either side of the strut location in the circumferential direction. The jump on the rear rotor was smaller, but was not quantified or compared with the front rotor.

Stürmer et al.[3] observed a similar jump in front rotor loading, and showed that it was largely independent of pylon blowing. In addition, they investigated the 'handedness' of the engine and pylon combination. That is, for the same engine, a horizontal pylon could be installed such that the front rotor moves down or up behind the pylon, as sketched in Fig. 1. They showed that the pylon affects the blade loading at $4^{\circ}$ AoA through changes to the effective incidence, such that the front blade peak load in the DBP sense is higher than that in the UBP sense. Stürmer et al. also provided some information about their geometry: they used a symmetric aerofoil, with $t / c=0.1$, whose leading edge was swept $15^{\circ}$ forwards. The pylon trailing edge was 0.15 front rotor diameters upstream of the front rotor plane of rotation.

A sharp increase in rotor loading behind the pylon was also observed at cruise, in simulations by Colin et al.[4] at $0^{\circ}$ AoA. This increase was explained as an impingement of the pylon wake causing a sudden change of incidence. Again, the loading gradually dropped as the blade rotated around the annulus. Colin et al. noticed additionally that the instantaneous loading was higher than the average loading when the instantaneous incidence was higher than the average incidence.

In this paper, high fidelity simulations of contra-rotating

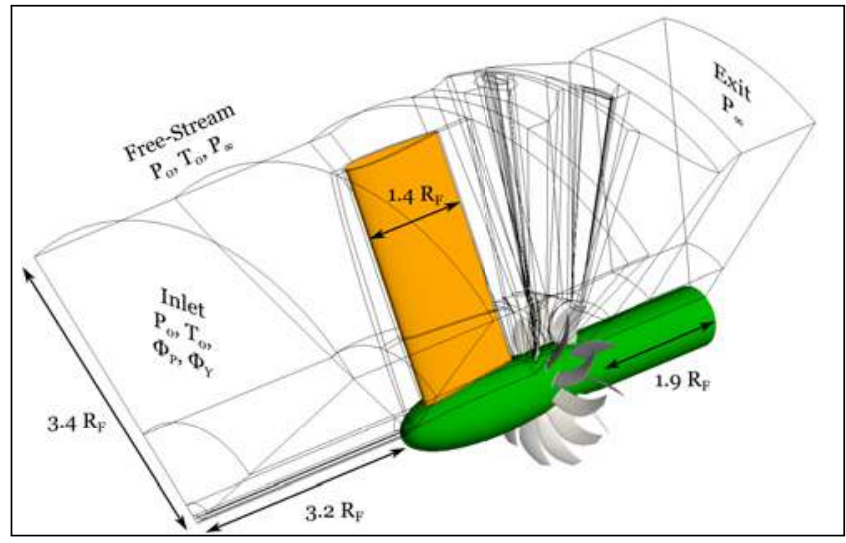

Figure 2. Computational domain showing pylon, rotors, and block boundaries.

open rotors developed previously by the authors [5] were extended to include an upstream pylon, at $0^{\circ}$ and $12^{\circ} \mathrm{AoA}$. The effect of the pylon on the incidence seen by the front rotor was linked to changes to the front rotor's work and loss. This modification of flow around the front rotor was then shown to affect the rear rotor incidence, work, and loss. In addition to examining simulation snapshots, these flow field differences were quantified by tracking streamlines through the time-averaged flow through both rotors.

\section{RIG TEST CASE}

The Zo8 Rig is a $1 / 7$ scale Airbus rig. The particular configuration studied here is one from a joint Airbus/Rolls-Royce test that used rotor blades designed by Rolls-Royce. More details are given by Paquet et al.[6] A take-off operating point was considered, and is summarised in Table 1. CFD simulations were performed at $0^{\circ}$ and $12^{\circ} \mathrm{AoA}$.

Table 1. Nominal Zo8 Key Parameters at Take-Off, for $0^{\circ}$ and $12^{\circ}$ AoA

\begin{tabular}{lcc}
\hline & Front Rotor & Rear Rotor \\
\hline Diameter $(\mathrm{m})$ & 0.610 & 0.518 \\
Blade Count, $Z$ & 12 & 9 \\
Stagger, $\gamma\left({ }^{\circ}\right)$ at $r / R_{F}=0.75$ & 51 & -39 \\
Advance Ratio, $\mathrm{J}$ & 1.09 & 1.49 \\
\hline Mach Number & \multicolumn{3}{c}{0.219} \\
\hline
\end{tabular}

The pylon used in the below simulations was representative. Its geometry was not taken from the Zo8 test campaign. It had a NACA 0012 section with a $20^{\circ}$ forward sweep and a fixed chord of $70 \%$ of the front rotor diameter. This pylon was placed upstream of the rotors in a 'pusher' configuration of the Zo8 Rig, such that the pylon trailing edge was $0.16 D_{F}$ upstream of the front rotor stagger change axis. 


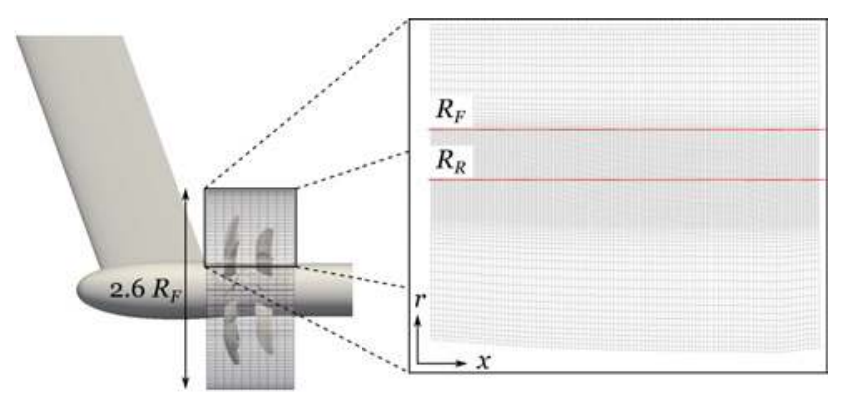

Figure 3. Relative domains and radial clustering of absoluteframe mesh.

\section{COMPUTATIONAL SETUP}

All CFD simulations presented in this paper have been performed with the Turbostream solver [7], which is based on a previous solver called TBLOCK by Denton [8]. A secondorder accurate finite-volume scheme operating on a multiblock structured grid is used for spatial discretisation, and in time, a second-order accurate implementation of the dual time-stepping (DTS) scheme [9] is employed. Scalar adaptive smoothing as per the JST scheme of Jameson et al.[10] is used to stabilise the numerical scheme. The one-equation Spalart-Allmaras turbulence model [11] with fully turbulent boundary layers is used for all simulations.

All the grids used here were created with Numeca AutoGrid 5 and include the full annulus and the complete bullet and pylon geometry with about $180 \times 10^{6}$ cells. This computational approach is an extension of previous simulations of the isolated engine by the authors [5], where the interaction between rotors was shown to be precisely resolved. The simulation domain and boundary conditions are illustrated in Fig. 2.

\section{POST-PROCESSING METHODS}

\subsection{Virtual Flow Tufts}

In order to show the flow on surfaces, an algorithm called Surface Line Integral Convolution (SLIC) was used. This technique generates random greyscale noise on the pixels representing surfaces, then displaces the pixels based on an underlying vector field. Here, pixels are displaced by the velocity field, to produce streaking patterns that follow the velocity field tangents. Such an approach is valid on instantaneous snapshots of a flow field, like a quiver plot or tufts, and produces images that look like streamlines. The algorithm for these 'virtual tufts' as implemented by ParaView is described by Burlen et al. [12], and is based on a combination of the algorithms described by Cabral and Leedom [13] and Laramee et al. [14]

\subsection{Front Rotor Tip Vortex Quantification}

The evolution of the front rotor's tip vortices was extracted from CFD by making several axial cuts between the rotors and calculating the vorticity vector on each slice. Finding cells with the locally highest vorticity on each slice then gave
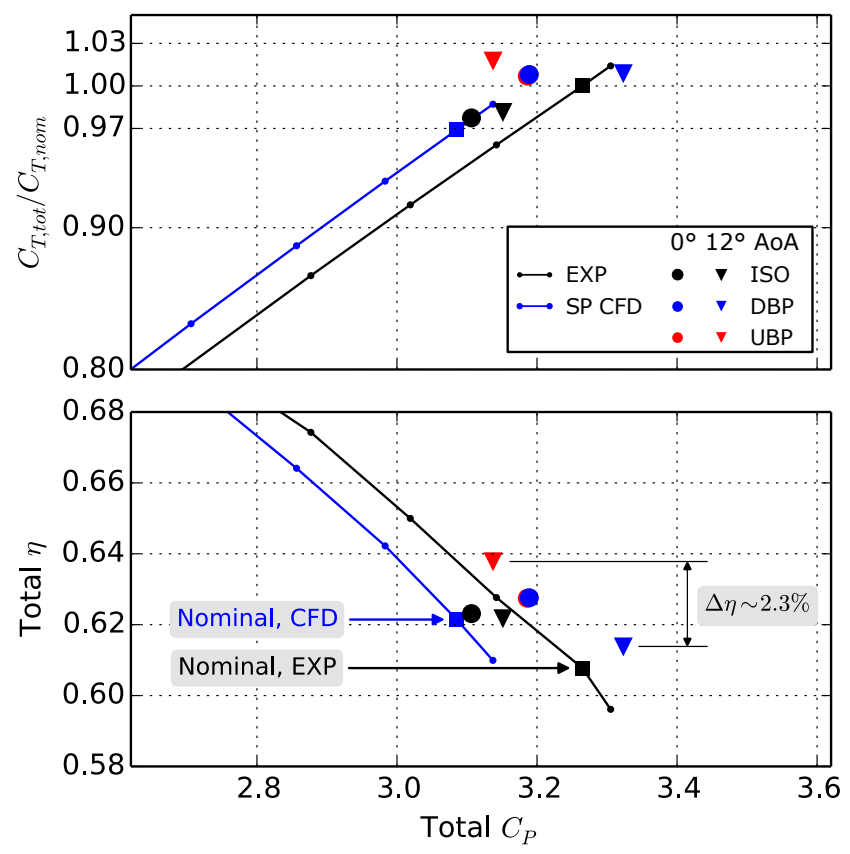

Figure 4. Engine performance parameters.

the locations of the vortex centres. The circulation of each of these vortices was then calculated by summing over cells inside an ellipse centred on the previously found highest vorticity cell, to approximate the integral $\Gamma=\iint_{S} \omega \cdot d S$. The ellipse size was varied around the annulus to always capture the vortex without including any of the vorticity from the wake. This process was repeated for three snapshots for each configuration, which were chosen at roughly equal spatial intervals. Figure 11 thus shows 36 data points per configuration at each axial cut.

\subsection{Time-Averaged Engine Flow}

In addition to time-accurate results, an analysis of the timeaveraged flow is presented. The average was calculated by first interpolating several full-annulus snapshots onto a mesh in the absolute frame (i.e. not rotating with either rotor), and then averaging over these interpolated snapshots. This absolute-frame mesh was a single axisymmetric block with hexahedral cells, which followed the Zo8 hub line, extended to $130 \%$ of the front rotor tip radius, and had 1200 circumferential points. In the radial direction, the mesh was clustered near the rotor tips. Figure 3 shows this clustering and the domain of the absolute-frame mesh relative to the rotors and pylon.

To reduce truncation error and aliasing in the average, 83 snapshots were used, such that the front rotor rotated about $5.78^{\circ}$ between snapshots and $480^{\circ}$ in total, and the rear rotor rotated $5^{\circ}$ between samples and $415^{\circ}$ in total. In the average, the rotors were ignored through the use of a gate function. That is, if a cell in the absolute-frame mesh happened to fall 'inside' a rotor blade at the interpolation stage, it was assigned the NaN value, which would then be ignored during the average. Lastly, streamlines were traced 

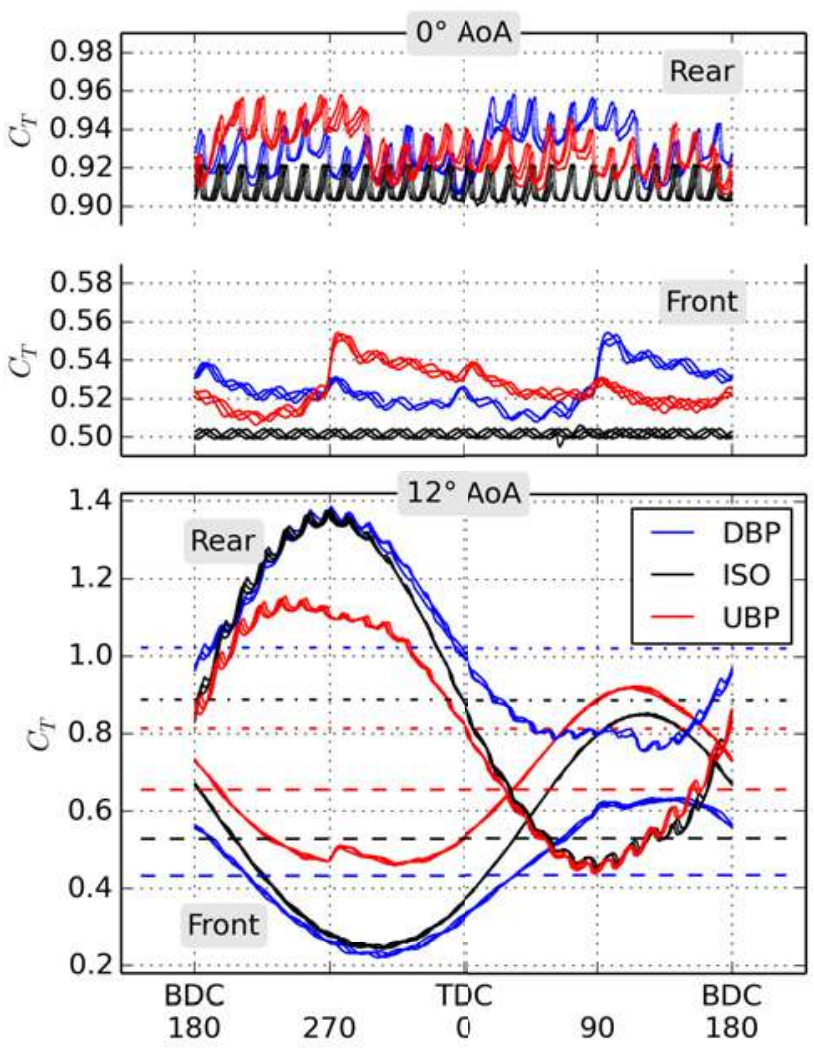

Figure 5. Variation of $C_{T}$ around the annulus.

through the averaged flow to calculate the distortion of the stream surface through the middle of the rotors. The seeds for these streamlines were provided in the absolute-frame mesh at $75 \%$ of the rotor tip radius at each rotor exit, and traced backwards to the rotor inlet. Some residual aliasing near the rear rotor was removed by smoothing in the circumferential direction over $6^{\circ}$ segments.

\section{RESULTS}

\subsection{Overall Performance}

Figure 4 shows the engine's operating line at nominal stagger, with the black line trend of experimental values closely matched by the blue line of single-passage steady CFD. This agreement in trends was previously shown by the authors [5] so the plot here is focussed on the nominal operating point. There are additional points in Fig. 4 representing timeaveraged values of full-annulus unsteady simulations. The black square marks the rig measurements at the nominal operating point, and the blue square marks the corresponding single-passage steady simulations. For the full-annulus cases, the circle and triangle markers represent time-averaged values at $0^{\circ}$ and $12^{\circ}$ AoA respectively. The $C_{T}$ values have been normalised by the nominal rig $C_{T}$, to highlight the spread of values: all simulations were within about $\pm 4 \%$ of the nominal rig $C_{T}$, so comparisons between the cases are meaningful.

The figure shows that at o ${ }^{\circ} \mathrm{AoA}, C_{T}$ for all unsteady simulations lies further up on the operating line, compared with
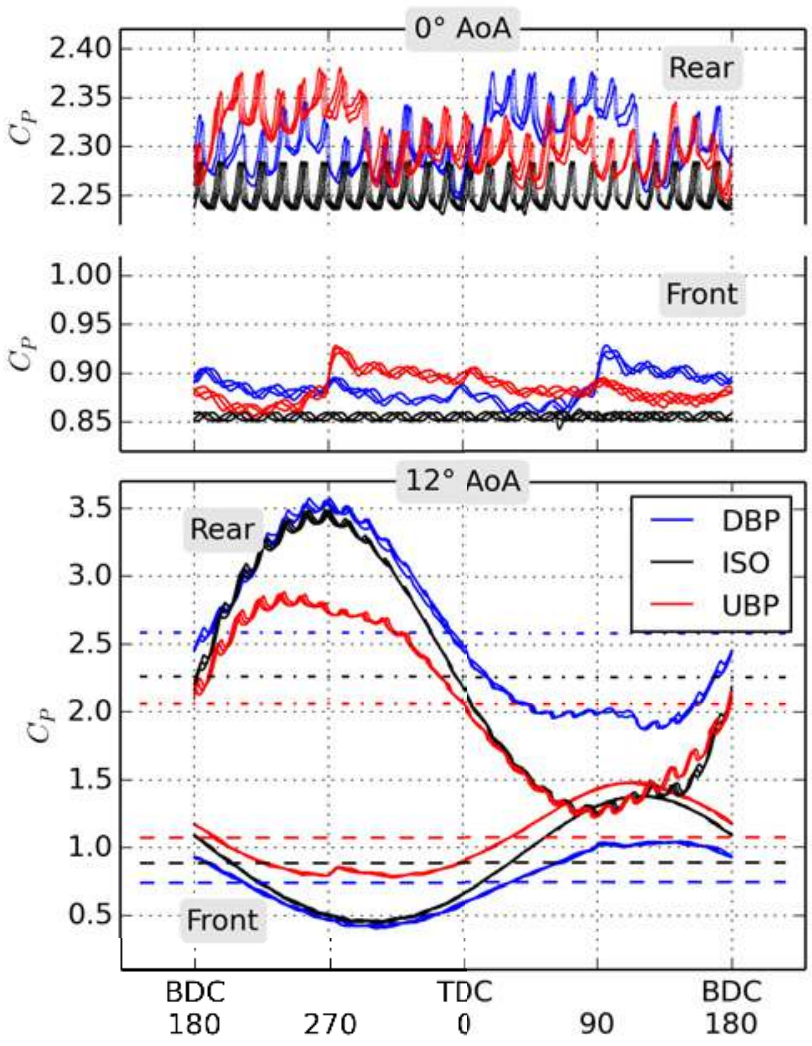

Figure 6. Variation of $C_{P}$ around the annulus.

the single-passage calculation at the nominal operating point, and the efficiency is of a comparable value. There is also no difference between the UBP and DBP configurations, to the extent that the DBP points almost coincide with the UBP points. At $12^{\circ} \mathrm{AoA}$, the puller and pusher configurations show no difference in efficiency, but the UBP and DBP configurations have $1 \%$ higher and lower $\eta$, respectively, relative to $0^{\circ}$ AoA.

The time-accurate variations in engine performance at both angles of attack are plotted in Figs. 5 and 6, where for each rotor, the variation in $C_{P}$ and $C_{T}$ of one blade over several rotor revolutions is plotted against $\theta$. The time-averaged values at $12^{\circ}$ AoA are plotted as dashed lines. The traces exhibit the relatively high-frequency rotor-rotor interaction, which does not repeat in the available simulation time, making the peaks and troughs precess in $\theta$. The once-per-rev features, due to AoA and the pylon, obviously do not precess.

The front rotor traces for the installed cases also show a jump at the location of the pylon, which is at $\theta=90^{\circ}$ in the DBP configuration, and at $\theta=270^{\circ}$ in the UBP configuration. The jump exists at both angles of attack, and the mechanism for it is a drop in $V_{x}$ in the pylon wake, which causes a local increase in the front rotor incidence. There is a similar jump in the rear rotor trace, though its magnitude is smaller than that seen on the front rotor, making it difficult to distinguish from the rotor-rotor interaction. It is clear that the magnitude of $C_{P}$ and $C_{T}$ variation due to the pylon wake is dwarfed by 
The influence of an upstream pylon on open rotor aerodynamics at angle of attack $-5 / 12$
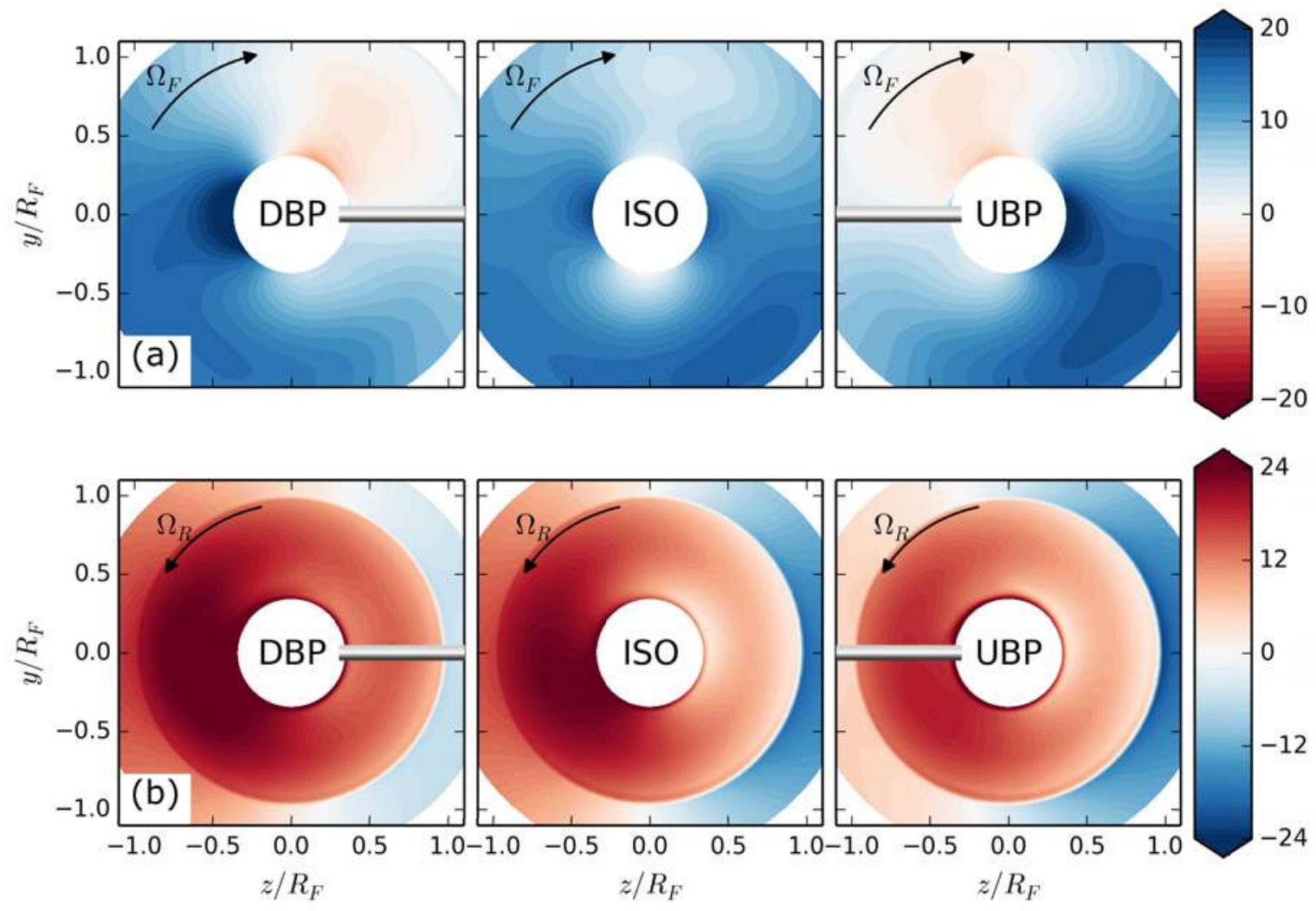

Figure 7. Contours of time-averaged flow: (a) front rotor inlet $\alpha_{l o c}$, and (b) rear rotor inlet whirl, at $12^{\circ}$ AoA.
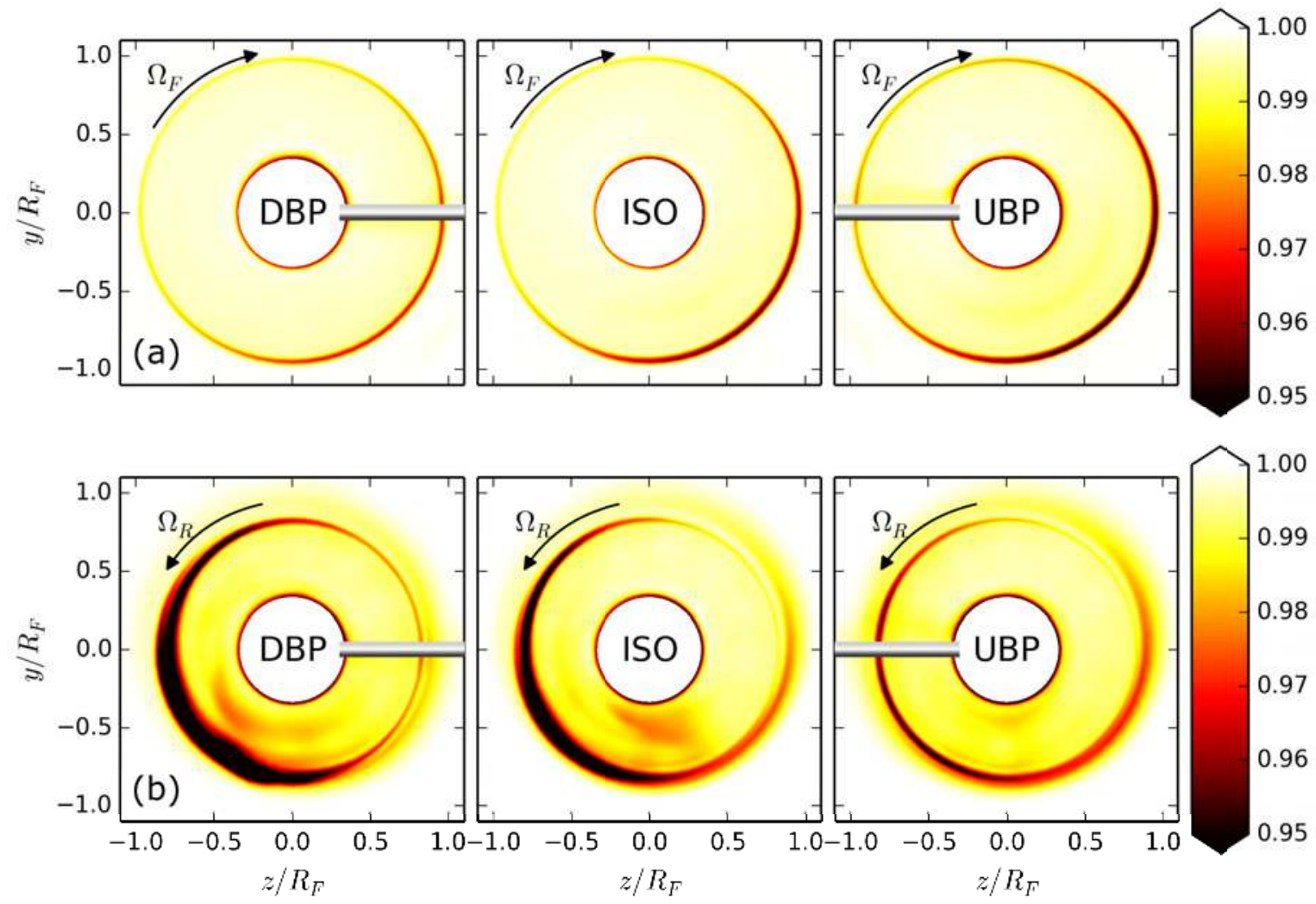

Figure 8. Contours of time-averaged entropy function at (a) front rotor exit, and (b) rear rotor exit, at $12^{\circ}$ AoA. 
Front Rotor
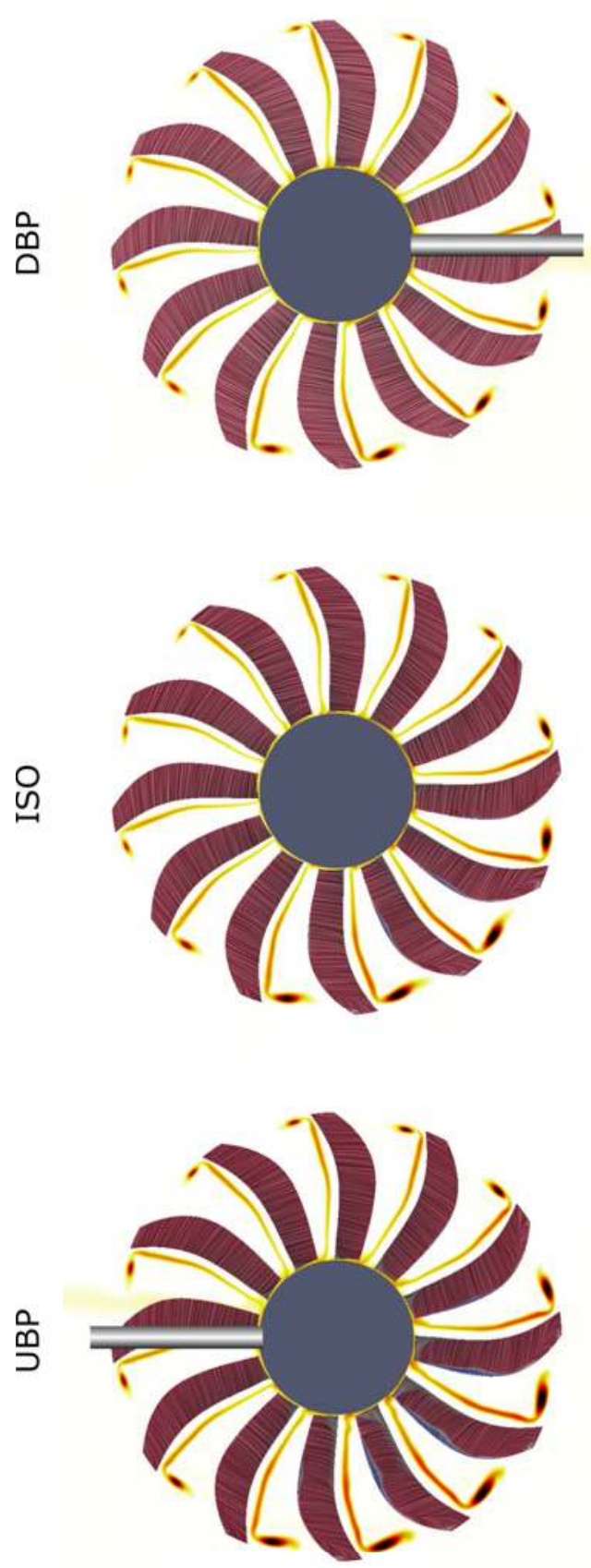

0.850

$-10$
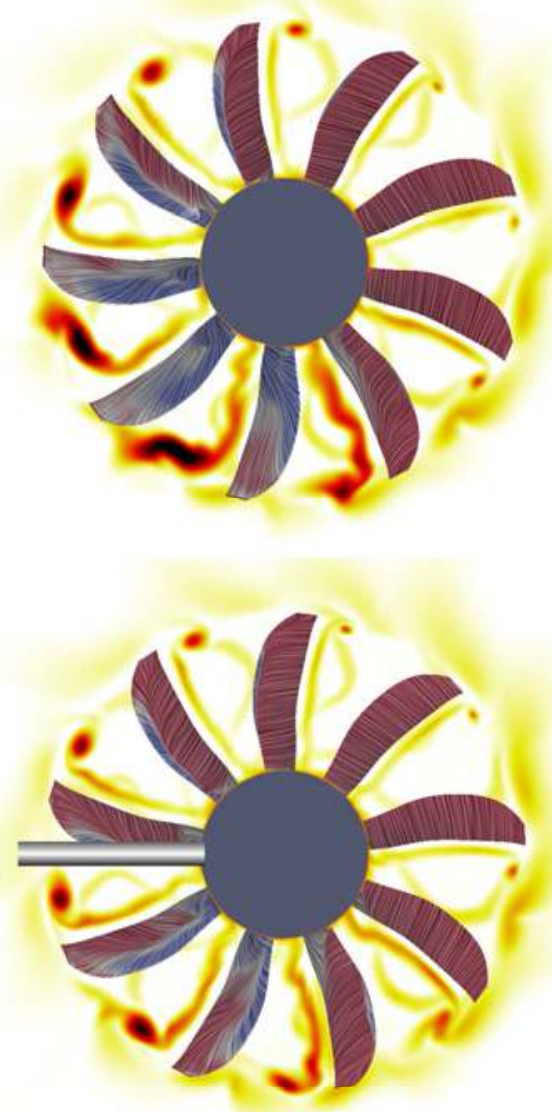

Figure 9. Contours of instantaneous entropy function at rotor exits, and virtual tufts on rotor suction surface, coloured by $V_{x}$, at $12^{\circ}$ AoA. Flow is into the page. 
the magnitude of variation linked to AoA.

At $12^{\circ} \mathrm{AoA}$, the figures also show a once-per-revolution 'modulation'. Compared with the traces for the isolated puller, the with-pylon traces appear clipped, especially for the rear rotor. This clipping shows that the pylon's influence does not extend to the entire annulus, and that the rotors return to behaving as though in an uninstalled configuration a short distance away from the pylon.

\subsection{Changes to Rotor Flow Field}

The flow behind the pylon can be further understood by inspecting the time-averaged flow through the rotors. As a consequence of the pylon having positive lift, flow is 'straightened' or turned towards the engine axis, with corresponding changes to rotor incidence relative to the uninstalled engine.

Firstly, Fig. 7 shows time-averaged contours of the 'local' angle-of-attack:

$$
\alpha_{l o c}=\arctan \left(\frac{V_{y}}{V_{x}}\right)
$$

at the front rotor inlet. It can be seen that the pylon straightens flow in the quadrant above it, to the extent that $\alpha_{l o c}$ even goes a few degrees negative, because streamtube contraction becomes dominant. The bullet straightens flow near the TDC and BDC in all cases, and accelerates it vertically near $y=0$. This vertical acceleration was also predicted by Gonzalez-Martino et al. [15], and is responsible for the dark blue patches in Fig. 7 and occurs only on the side opposite to the pylon for the installed cases. These variations in $\alpha_{l o c}$ act to increase the mean and peak incidences onto the front rotor in the UBP case, and decrease them in the DBP case.

The influence of the pylon extends to the rear rotor, as shown in Fig. 7 (b), through contours of whirl at the rear rotor inlet. The whirl angle here is positive in the $\theta$ direction, i.e. opposite to the rear rotor rotation. Looking first at the whirl beyond the rear rotor radius, it can be seen in the installed cases that the magnitude of whirl is largest on the side opposite to the pylon, becase the flow on that side has not been straightened. Note that the straightening appears as a reduction in whirl in the UBP case, and an increase in the DBP case. The same happens within the rear rotor radius, so the peak whirl is lowest for the UBP case, and the minimum whirl is highest for the DBP case. The DBP peak whirl is higher, by a few degrees, than the isolated peak whirl, which is linked to flow accelerating vertically as it spills around the bullet.

The corresponding time-averaged contours of entropy function $^{1}$ (Fig. 8) show that the UBP case has the highest front rotor loss. The contours also show a thin pylon wake where very little entropy has been generated, as should be expected from the clean flow over the pylon. The entropy function contours at the exit of the rear rotor show the effect of the above changes in whirl: the loss is lowest in the UBP configuration.

${ }^{1}$ Defined as $e^{-\Delta s / \bar{R}}$, where $\Delta s$ is the change in specific entropy relative to the inlet and $\bar{R}$ is the specific gas constant for dry air.
An overview of the changes to the flow through the rotors is provided by Fig. 9 . It contains snapshots, at $12^{\circ}$ AoA, of both rotors with virtual tufts, and contours of entropy function at the rotor exits. Compared with the uninstalled engine (ISO), the flow on the rear rotor in the DBP case is worse. The hub separation and leading edge bubble are no longer suppressed in the $0^{\circ}$ to $90^{\circ}$ quadrant, and more loss is swept to the tip in the opposite quadrant, as evidenced by the larger patches of black. On the other hand, the flow on the rear rotor in the UBP case has improved, because in the lower left quadrant, instead of radial (gray) or reversed (blue) flow on the whole blade, there is reversed flow only in the lower $25 \%$ of the blade span, and in the leading edge separation. This improvement is also reflected in the contours of entropy function at the rotor exit. The circumferential extents of the worst flow are approximately the same across configurations.

The changes in flow structure and entropy generation between DBP, UBP and uninstalled cases for the front rotor are smaller. When rotating DBP, the front rotor tip vortices are smaller than when rotating UBP, which is related to the lower average and peak loading in the former. In the UBP case, blades in the lower right quadrant have leading edge bubbles that extend about twice as far in the chord-wise and span-wise directions than on the isolated engine. In this quadrant, blades have also acquired hub separations, as evidenced by the patches of gray, and indicate that the hub incidence is higher than on the other two cases. This plot suggests that there is further scope to improve the efficiency in the UBP configuration: by increasing the stagger of both rotors to reduce incidence and diminish the separations, while increasing the rotor speeds to recover thrust, similar to the strategy of Zachariadis et al.[16]

The straightening effect of the pylon on the front rotor tip vortex trajectories is quantified in Figs. 10 and 11 . The first of these shows the radial spread of the tip vortex centres at several axial locations up to the rear rotor stagger change axis (SCA). The spread is caused by the engine AoA, with the most radially outwards vortex expected to be near TDC, and the

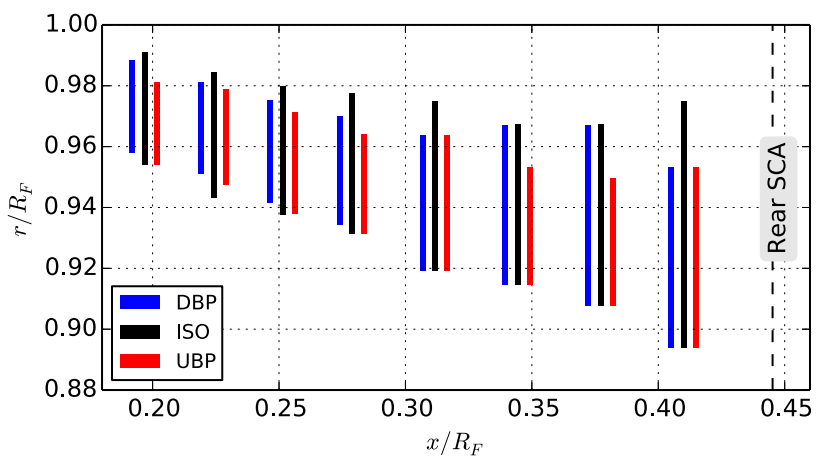

Figure 10. Radial and axial variation of front rotor tip vortex cores. Cuts for all cases were made at the same axial locations, at $12^{\circ} \mathrm{AoA}$, but are staggered for clarity. Rear rotor stagger change axis is marked. 

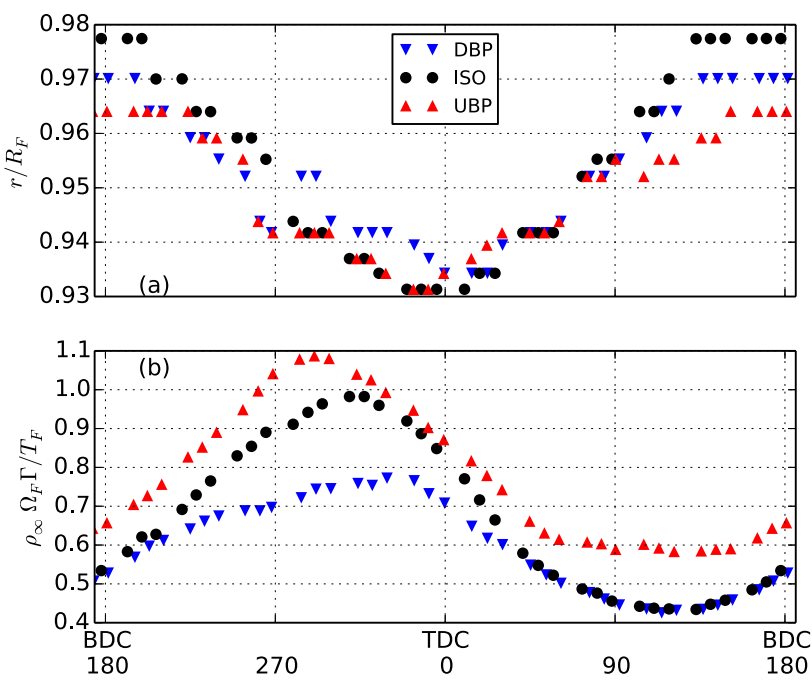

Figure 11. Circumferential variation of (a) radial location of vortex core, and (b) vortex circulation, at $x \simeq 0.14 D_{F}$, for $12^{\circ}$ AoA.

most radially inwards one near BDC. The pylon has limited the radially outwards advection of the vortices up to about $0.32 R_{F}$. When the front rotor moves UBP, the tip vortex that ends up near TDC at a given cut will have travelled through the straightened zone above and behind the pylon (see Fig. 7), so its radial migration is more severely limited than in the BDC configuration, where the highest tip vortex does not spend as much time in the straightened zone. Beyond $0.32 R_{F}$, and closer to the rear rotor, the influence of the pylon appears to be diminished for the DBP case.

The circumferential variation of the vortex locations at $0.28 R_{F}$ is plotted in Fig. 11, where the 36 vortices are clearly visible. The variation in circulation is also plotted. The latter exhibits clipping similar to the $C_{T}$ plots, because tip vortex strength is directly linked to the lift on an aerofoil.

\subsection{Average Streamlines at Mid-Span}

The discussion is now turned to the detailed work and loss variations of each rotor as calculated from average streamlines. This section uses streamlines traced through the timeaveraged mesh to quantify changes in incidence, loss and work for both rotors. In the subsequent figures, quantities such as incidence and Mach number are computed at the end-points of the streamlines, i.e. at the rotor inlets. Other quantities are calculated per streamline, and plotted against the inlet $\theta$. These plots only show variations from streamlines seeded at the rotor exits at mid-span.

In Fig. 12 one can see spikes in incidence and corresponding drops in Mach number behind the pylon. It is this combination that causes the jump in loading shown in Figs. 5 and 6. The straightening effect of the pylon is also apparent: the incidence is clipped in a similar manner to the loading, though the velocity deficit in the pylon wake in the DBP case keeps the peak incidence at almost the same value as for
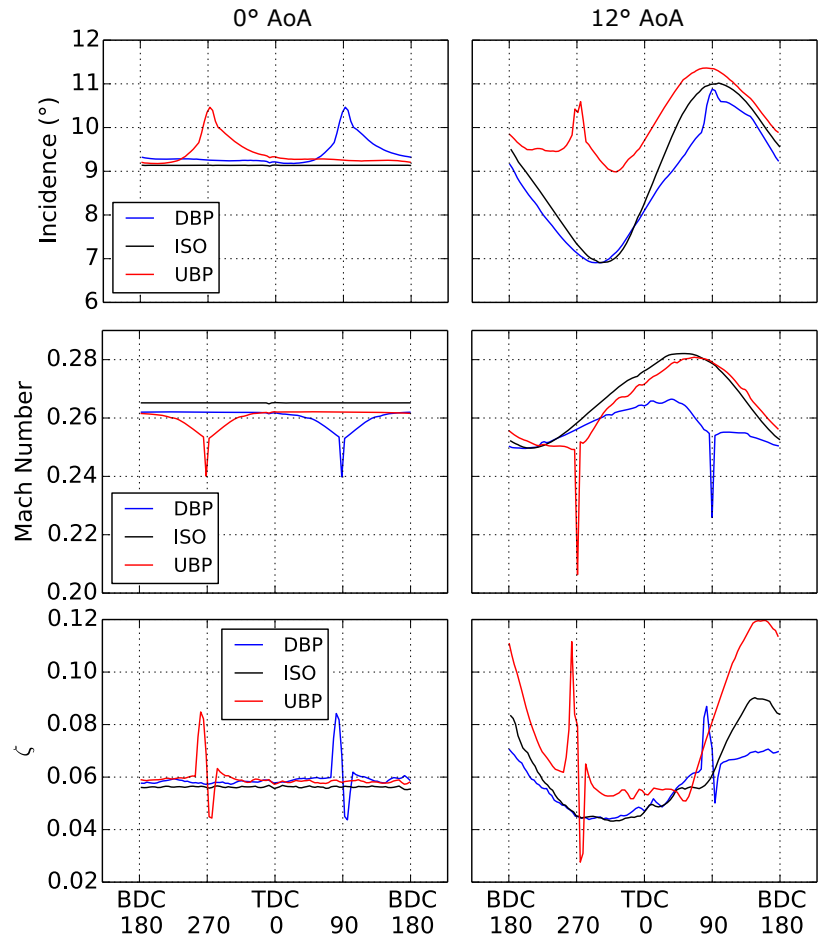

Figure 12. Annular variation of incidence, inlet Mach number, and $\zeta$ for front rotor mid-span

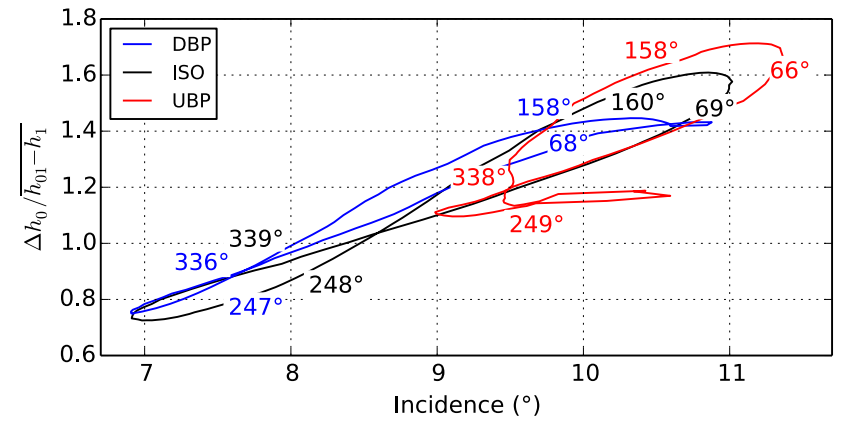

Figure 13. Work and incidence on time-averaged streamlines, front rotor mid-span, at $12^{\circ} \mathrm{AoA}$.
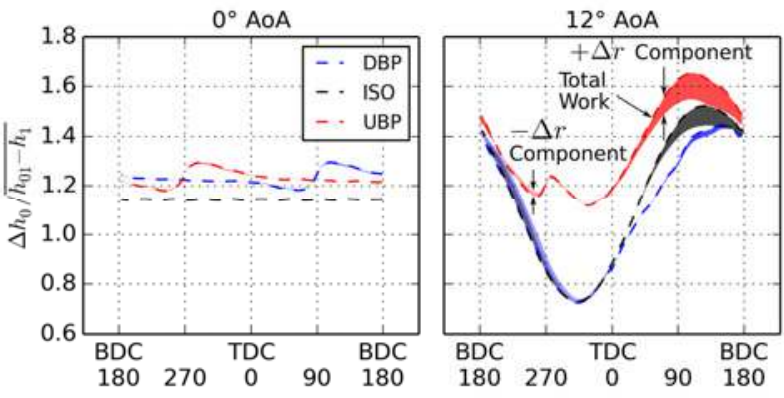

Figure 14. Effect of change of radius on front rotor work. 


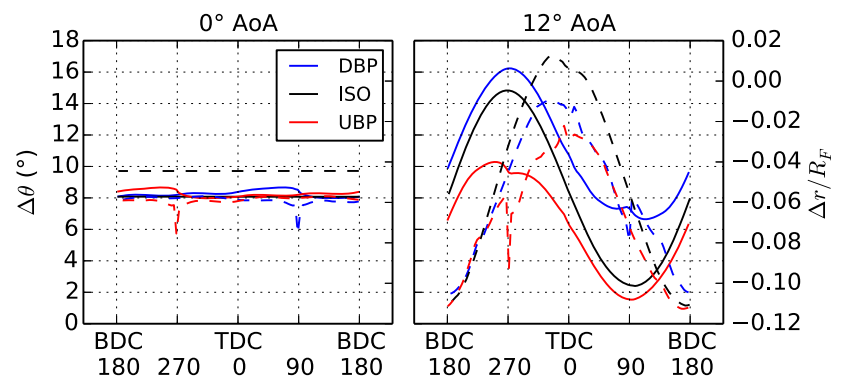

Figure 15. Annular variation of $\Delta \theta$ (solid lines) and $\Delta r$ (dashed lines), front rotor mid-span.

the isolated case. The Mach number at $12^{\circ}$ AoA for the DBP case is lower, on average, than the other two cases because the front rotor loading is lower in the same quadrant as the pylon drag, meaning the two phenomena reinforce instead of cancel out. The 'free-stream' Mach number in the installed cases at $0^{\circ} \mathrm{AoA}$ is about $1.2 \%$ lower than the isolated value, and is a consequence of the longer bullet.

It is also apparent that the large change in $\zeta$ between cases is not explained simply by incidence and Mach number, because the peak incidences are within $0.5^{\circ}$ of each other, and the Mach number only varies between 0.21 and 0.28 , which is not expected to significantly affect loss. The plots of loss show positive and negative spikes at the position of the pylons, of which the positive ones are meaningful, but the negative ones are not. The latter are thought to be caused by small errors in the tracing of the streamlines, i.e. when a streamline starts in the pylon wake but ends outside of it.

Figure 13 shows the variation of work with incidence as calculated from the mid-span streamlines. Unlike the loss, the maxima in work and incidence coincide. This plot shows that the variation in work is almost fully attributable to the variation in incidence, with the least hysteresis for the DBP case, and the largest for the UBP case. That is, for a given incidence, the difference in work across the hysteresis loop is at most $5 \%$ for the DBP case, and about $10 \%$ for the UBP case, ignoring the wake.

The work done on each streamline is plotted in Fig. 14, with the contribution of the change of radius term highlighted. The work done on each streamline can be split into $\Delta r$ and $\Delta V_{\theta}$ components as follows:

$$
\begin{aligned}
h_{02}-h_{01} & =\Omega r_{2} V_{\theta 2}-\Omega r_{1} V_{\theta 1} \\
& =\Omega r_{2}\left(V_{\theta 1}+\Delta V_{\theta}\right)-\Omega\left(r_{2}-\Delta r\right) V_{\theta 1} \\
& =\Omega r_{2} \Delta V_{\theta}+\Omega V_{\theta 1} \Delta r
\end{aligned}
$$

The variation of streamline work around the annulus is plotted in Fig. 14, with the contribution of the $\Delta r$ highlighted. Although a positive $\Delta r$ component is usually associated with 'free' work from an increase in radius, in this case Fig. 15 shows that the change in radius is always negative, meaning that the second term in Eq. (1) can only be positive when $V_{\theta 1}$ is negative. This velocity is negative at the front rotor inlet for $\theta$ between $o^{\circ}$ and $180^{\circ}$ because of the engine AoA, so the

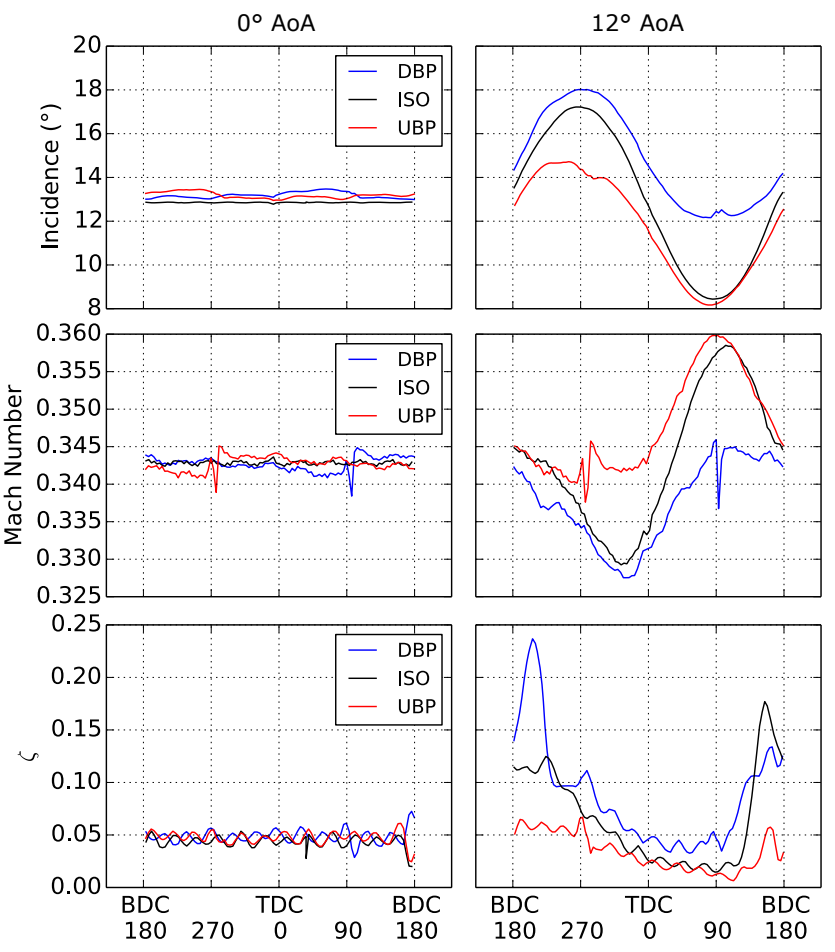

Figure 16. Annular variation of incidence, inlet Mach number, and $\zeta$ for rear rotor mid-span

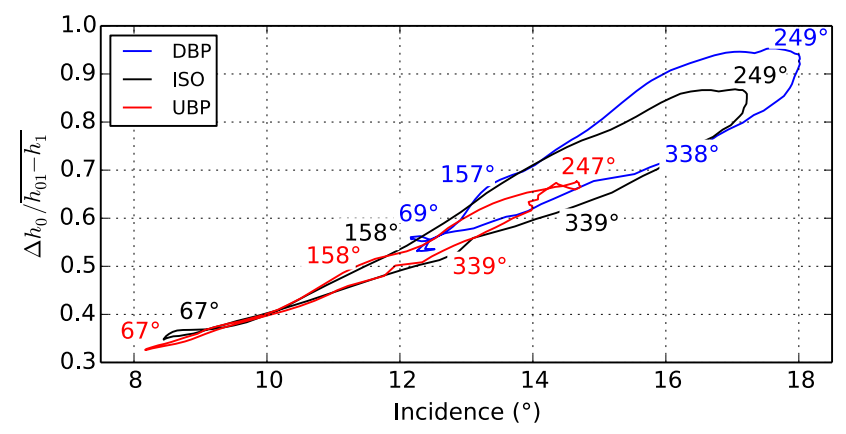

Figure 17. Variation of work and $\zeta$ with incidence, rear rotor mid-span, at $12^{\circ}$ AoA.
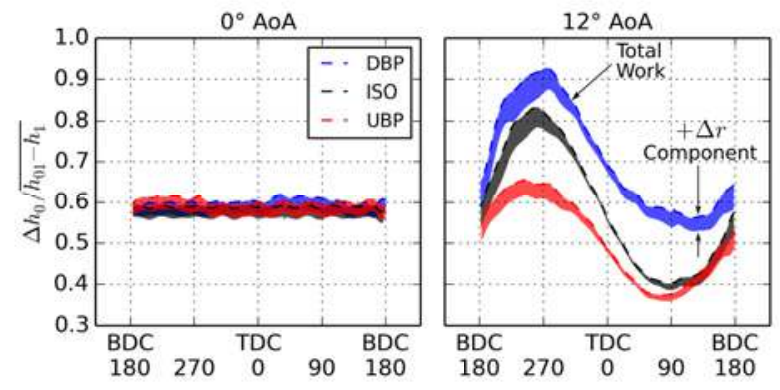

Figure 18. Effect of change of radius on rear rotor work. 


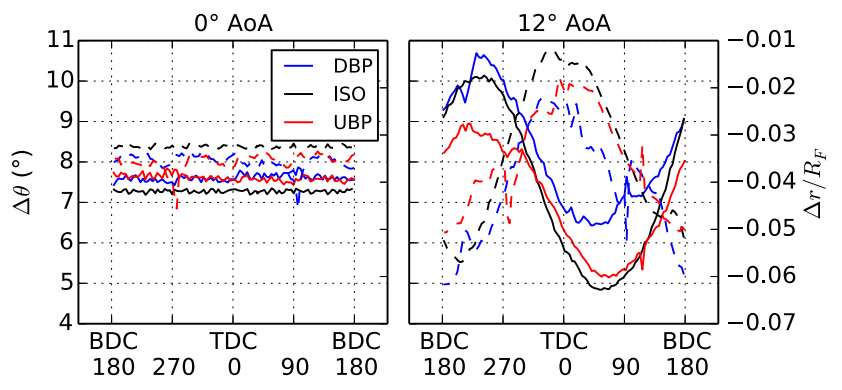

Figure 19. Annular variation of $\Delta \theta$ (solid lines) and $\Delta r$ (dashed lines), rear rotor mid-span

second term represents 'free' work due to AoA.

The same plots for the rear rotor, Figs. 16 and 17, show a similar story. The labels DBP and UBP still refer to the rotation of the front rotor, so the rear rotor moves up behind the pylon in the DBP case and vice versa. The variations in incidence and Mach number clearly exhibit clipping, which is caused by a combination of the circumferential variation in both rotors' loading and the pylon's drag. The variation in loss is more severe, with the highest loss, in the DBP case, being nearly twice the highest loss for the front rotor. There is still a phase difference between the incidence and $\zeta$.

The $\Omega V_{\theta 1} \Delta r$ component of work for the rear rotor (Fig. 18) is always positive for the rear rotor, and as a fraction of the total work, can be up to $10 \%$. Again, the streamline deformation plot, Fig. 19, shows that $\Delta r$ is always negative, meaning that this component shows the 'free' work due to engine AoA, because the rear rotor inlet whirl is also always negative.

\subsection{Pressure Harmonics at Mid-Span}

Although the effect of the pylon's viscous wake on engine noise is not the focus of the present article, it is addressed indirectly here for comparison with the effect of the pylon potential field. The acoustic implications were quantified through a comparison of the harmonics of pressure fluctuations on each rotor's suction surface near the leading edge, at approximately mid-span. The full noise predictions would, obviously, require contributions from the rest of the blade, and would need to account for blade stacking and rotor velocity.

Figure 20 shows that at $0^{\circ} \mathrm{AoA}$, the rotor-alone tones are around 4 orders of magnitude louder when the engine is installed behind a pylon, regardless of pylon orientation. Rotor-alone harmonics up to about the 81st are plotted, and are all louder than the first four interaction tones. At $12^{\circ}$ AoA, there is an insignificant increase in the magnitudes of these harmonics for the installed cases. As an aside, some side-bands can be seen in the spectrum of the isolated configuration, as marked by the clustered gridlines, but these are dwarfed by tones at multiples of the shaft speed in the installed spectra.

On the rear rotor (Fig. 21), the pylon's influence is reduced, but not negligible: installation causes an increase of at most two orders of magnitude in rotor-alone tones. The loudest harmonics here are the rotor-rotor interaction tones, both at $0^{\circ}$ and $12^{\circ}$ AoA. Similar to the front rotor, the change in AoA causes a change in harmonic magnitude smaller than the increase due to installation, with the only exception being the first few tones at $12^{\circ}$ AoA. The reduction of the pylon wake's influence can alternately be seen by first comparing isolated cases only: at $0^{\circ} \mathrm{AoA}$, the rear rotor fluctuations are generally higher, and the rotor interaction tones dominate. At $12^{\circ} \mathrm{AoA}$, the rear rotor fluctuations are mostly higher. In the installed case, the rotor fluctuations are dominated by the pylon wake, so the rear rotor fluctuations are generally smaller than those on the front rotor, but larger at o AoA than the isolated case. The rotor interaction tones appear to be insensitive to the pylon.

The fluctuations in static pressure on the rear rotor in the UBP and DBP cases, as plotted in Fig. 21, appear similar in magnitude despite the cleaner flow in the UBP configuration for the following reasons. Firstly, even in the UBP case, there is a leading edge separation on the down-stroke, so the fluctuations at the probe location are reasonably large. Further, the log scale of the y-axis, intended to show the large effect of installation, obscures the difference between the two pylon configurations. For example, the DBP fluctuation is nearly twice as large as the UBP fluctuation at the rear rotor shaft frequency. The magnitudes at higher frequencies depend on the circumferential extent of separation, and more generally, the 'shape' of the circumferential variation in pressure, so the differences between UBP and DBP are not straightforward to estimate, even qualitatively.

\section{CONCLUSIONS}

A high fidelity computational approach for the simulation of an open rotor engine installed behind a pylon and operating at angle of attack has been established. Novel post processing techniques including time averaging the flow field through rotors have also been applied.

For operation at angle of attack, a pylon upstream of an open rotor turns the oncoming flow towards the engine centreline, changing the effective flow angles onto the rotor blades. If the front rotor is rotating such that it is moving upwards behind the pylon, this leads to higher incidence angles and increased loading on the front rotor but lower incidence angles and loadings on the rear rotor. As a result, increased tip vortices and wakes from the front rotor are generated, but a more uniform flow is present within the rear rotor. In contrast, if the front rotor is moving downwards behind the pylon, its flow field is more uniform, but the loss in the rear rotor increases and the rear rotor wakes and vortices become large and highly non-uniform.

The orientation of the pylon was found to have a significant effect on overall open rotor efficiency because changes caused by the pylon to front rotor losses were smaller than the changes in the rear rotor losses. In general, for any contrarotating open rotor, it should be possible to find the pylon orientation that gives the highest efficiency when operating 

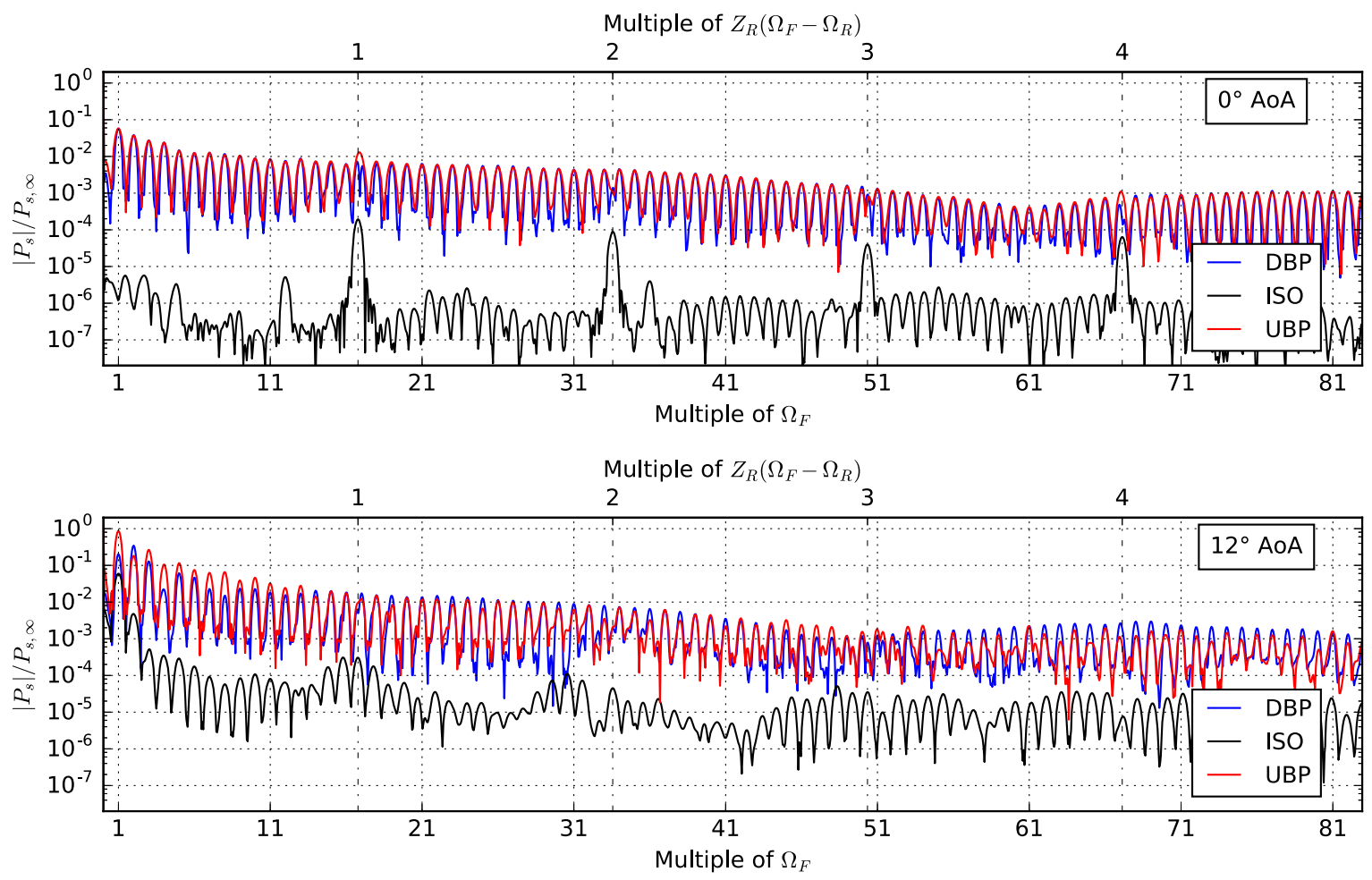

Figure 2o. FFT of static pressure at front rotor mid-span for $0^{\circ}$ and $12^{\circ} \mathrm{AoA}, r / R_{F}=0.75, x / c=0.1$
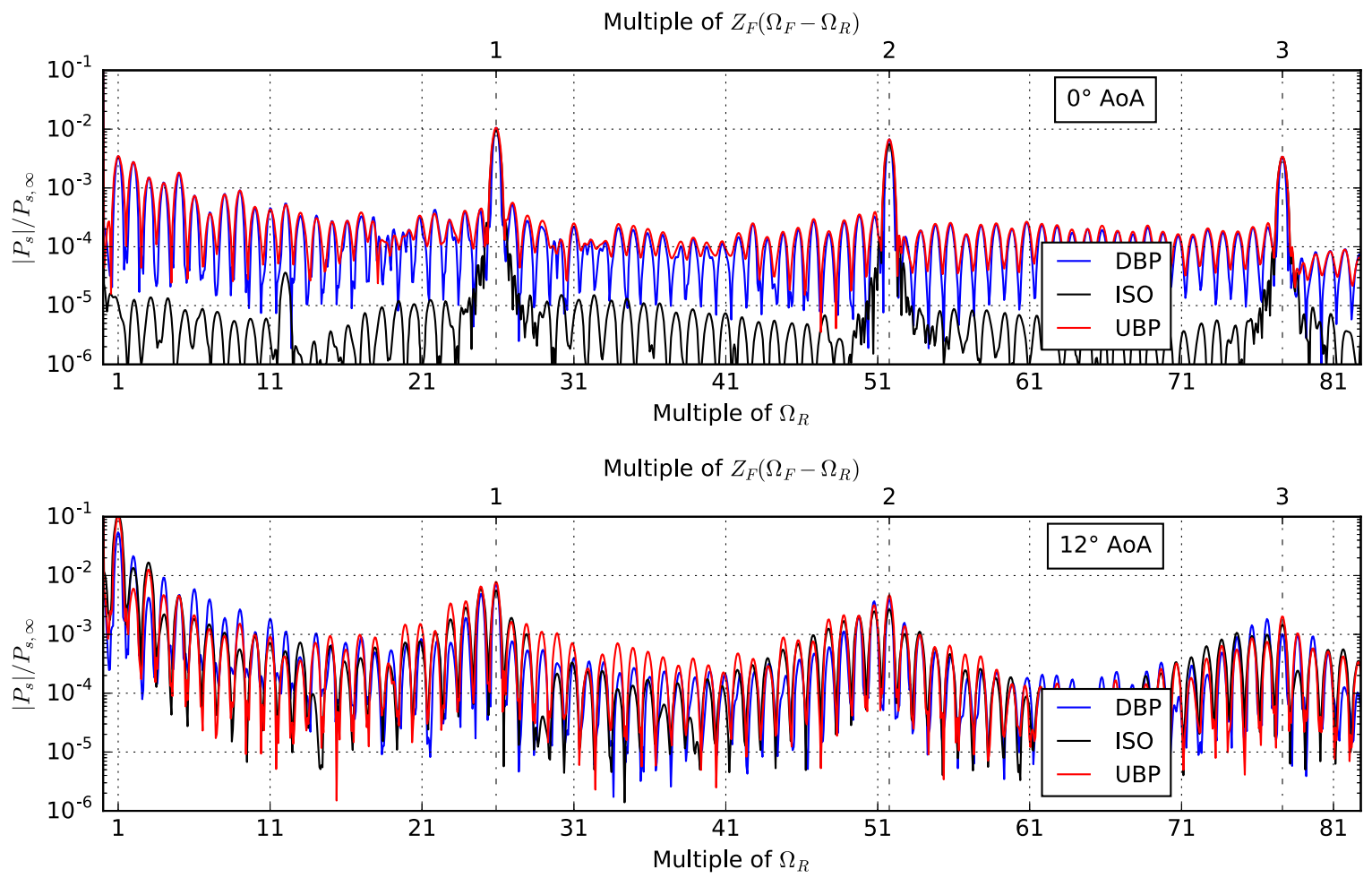

Figure 21. FFT of static pressure at rear rotor mid-span for $o^{\circ}$ and $12^{\circ} \mathrm{AoA}, r / R_{R}=0.75, x / c=0.1$ 
at angle of attack.

Time-averaging the flow through the rotors shows that the variation in work done on streamlines depends strongly on incidence. There is also a smaller contribution from the effects of radius change.

\section{ACKNOWLEDGMENTS}

The authors would like to thank Rolls-Royce plc, SAGE ITD (part of the Clean Sky JTI), and EPSRC for funding. Thanks also to Rolls-Royce plc for permission to publish the data. This work used the Wilkes GPU cluster at the University of Cambridge High Performance Computing Service, provided by Dell Inc., NVIDIA and Mellanox, and part funded by STFC with industrial sponsorship from Rolls-Royce and Mitsubishi Heavy Industries. The authors also gratefully acknowledge Turbostream Ltd. and Tobias Brandvik for their support with the solver setup and others at the Whittle Laboratory for their insight.

\section{REFERENCES}

[1] J. Ricouard, E. Julliard, M. Omaïs, V. Regnier, Anthony B. Parry, and S. Baralon. Installation effects on contrarotating open rotor noise. In 16th AIAA/CEAS Aeroacoustics Conference, 2010. AIAA 2010-3795.

[2] Ronan Boisard, Gregory Delattre, and Fabrice Falissard. Computational fluid dynamics as a support to counterrotating open-rotor wind-tunnel test analysis. Fournal of Aircraft, 51(2):614-628, March-April 2014.

[3] Arne Stürmer, Jianping Yin, and Rinie Akkermans. Progress in aerodynamic and aeroacoustic integration of CROR propulsion systems. The Aeronautical fournal, 118:1137-1158, October 2014.

[4] Yann Colin, Fabien Wlassow, Bastien Caruelle, Thomas Nodé-Langlois, Magdi Omaïs, Pierre Spiegel, and Anthony B. Parry. Installation effects on contra-rotating open rotor noise at high-speed. In 2oth AIAA/CEAS Aeroacoustics Conference, June 2014.

[5] Nishad G. Sohoni, Cesare A. Hall, Tobias Brandvik, and Anthony B. Parry. Prediction and measurement of unsteady blade surface pressures on an open rotor. In Proceedings of ASME Turbo Expo, 2015. GT2015-42334.

[6] Cédric Paquet, Emmanuel Julliard, Julien Ricouard, and Pierre Spiegel. Zo8: low-speed aero-acoustic experimental characterization of open rotor installation on aircraft. In 2oth AIAA/CEAS Aeroacoustics Conference, 2014. AIAA 2014-2747.

[7] Tobias Brandvik and Graham Pullan. An accelerated ${ }_{3} \mathrm{D}$ Navier-Stokes solver for flows in turbomachines. In Proceedings of ASME Turbo Expo, 2009. GT2009-60052.

[8] John D. Denton. An improved time-marching method for turbomachinery flow calculation. Fournal of Engineering for Gas Turbines and Power, 105(3):514-521, 1983.
[9] Antony Jameson. Time dependent calculations using multigrid, with applications to unsteady flows past airfoils and wings. In AIAA 1oth Computational Fluid Dynamics Conference, June 1991. AIAA 91-1596.

[10] Antony Jameson, Wolfgang Schmidt, and Eli Turkel. Numerical solutions of the Euler equations by finite volume methods using Runge-Kutta time-stepping schemes. In AIAA 14th Fluid and Plasma Dynamics Conference, 1981. AIAA 81-1259.

[11] P. R. Spalart and S. R. Allmaras. A one-equation turbulence model for aerodynamic flows. Recherche Aerospatiale, 1:5-21, 1994.

[12] B. Loring. Line integral convolution in ParaView, 2013. https : //web . archive.org/web/20160606 023821/http: //Www.paraview.org/Wiki/ ParaView/Line_Integral_Convolution.

[13] Brian Cabral and Leith Casey Leedom. Imaging vector fields using line integral convolution. In Proceedings of the 2oth annual conference on computer graphics and interactive techniques - SIGGRAPH '93. Association for Computing Machinery (ACM), 1993.

[14] R.S. Laramee, B. Jobard, and H. Hauser. Image space based visualization of unsteady flow on surfaces. In IEEE Transactions on Ultrasonics, Ferroelectrics and Frequency Control. Institute of Electrical and Electronics Engineers (IEEE), 2003.

[15] I. Gonzalez-Martino, B. Francois, and B. Rodriguez. A numerical insight into contra-rotating open rotor inplane loads. Mechanics \& Industry, 15(1):19-28, April 2014 .

[16] Alexios Zachariadis, Cesare A. Hall, and Anthony B. Parry. Contra-rotating open rotor operation for improved aerodynamics and noise at takeoff. In Proceedings of ASME Turbo Expo, 2011. 